\title{
Neutrino interaction with background matter in a noninertial frame
}

\author{
Maxim Dvornikov* \\ Physics Faculty, National Research Tomsk State University, 36 Lenin Ave., 634050 Tomsk, \\ Russia; \\ Pushkov Institute of Terrestrial Magnetism, Ionosphere and Radiowave Propagation \\ (IZMIRAN), 142190 Troitsk, Moscow, Russia; \\ Institute of Physics, University of São Paulo, CP 66318, CEP 05315-970 São Paulo, SP, Brazil \\ *E-mail: maxdvo@izmiran.ru
}

\begin{abstract}
We study Dirac neutrinos propagating in rotating background matter. First we derive the Dirac equation for a single massive neutrino in the noninertial frame, where matter is at rest. This equation is written in the effective curved space-time corresponding to the corotating frame. We find the exact solution of the Dirac equation. The neutrino energy levels for ultrarelativistic particles are obtained. Then we discuss several neutrino mass eigenstates, with a nonzero mixing between them, interacting with rotating background matter. We derive the effective Schrödinger equation governing neutrino flavor oscillations in rotating matter. The new resonance condition for neutrino oscillations is obtained. We also examine the correction to the resonance condition caused by the matter rotation.
\end{abstract}

Keywords: massive and mixed neutrinos, Dirac equation in curved space-time, exact solution, neutrino oscillations in matter, noninertial effects, rotation

\section{Introduction}

Nowadays it is commonly believed that neutrinos are massive particles and there is a nonzero mixing between different neutrino generations. These neutrino properties result in transitions between neutrino flavors, or neutrino oscillations. It is also known that various external fields, like electroweak interaction of neutrinos with background fermions, neutrino electromagnetic interaction, and neutrino interaction with a strong gravitational field, can also influence the process of neutrino oscillations.

As shown in Ref. 1, noninertial effects in accelerated and rotating frames can affect neutrino propagation and oscillations. The consideration of the reference frame rotation is particularly important for astrophysical neutrinos emitted by a rapidly rotating compact star, e.g., a pulsar. For example, the possibility of the pulsar spin down by the neutrino emission and interaction with rotating matter was recently discussed in Ref. 2. It should be noted that besides elementary particle physics, various processes in noninertial frames are actively studied in condensed matter physics. For instance, the enhancement of the spin current in a semiconductor moving with an acceleration was recently predicted in Ref. 3 .

In the present work we summarize the results of Ref. 4, where the neutrino in- 
teraction with background matter in a rotating frame was studied. We assume that neutrinos can interact with background fermions by means of electroweak forces. We also take that neutrino mass eigenstates are Dirac particles. In our treatment we account for the noninertial effects since we find the exact solution of a Dirac equation for a neutrino moving in the curved space-time with a metric corresponding a rotating frame.

This work is organized in the following way. In Sec. 2, we start with the brief description of the neutrino interaction with background matter in Minkowski spacetime. We consider matter moving with a constant velocity and discuss both neutrino flavor and mass eigenstates. Then, in Sec. 3. we study background matter moving with an acceleration. The Dirac equation, including noninertial effects, for a neutrino interacting with such matter is written down in a frame where matter is at rest. In Sec. 3.1, we solve the Dirac equation and find the neutrino energy spectrum for ultrarelativistic neutrinos moving in matter rotating with a constant angular velocity. Then, in Sec.4, we apply our results for the description of neutrino oscillations in rotating background matter. The effective Schrödinger equation governing neutrino oscillations is derived and the new resonance condition is obtained. We consider how the matter rotation can affect the resonance in neutrino oscillation in a realistic astrophysical situation. Finally, in Sec. 5. we summarize our results.

\section{Neutrino interaction with background matter}

In this section we describe the interaction of different neutrino flavors with background matter in a flat space-time. We discuss a general case of matter moving with a constant mean velocity and having a mean polarization. Then we consider the matter interaction of neutrino mass eigenstates, which are supposed to be Dirac particles.

The interaction of the neutrino flavor eigenstates $\nu_{\alpha}, \alpha=e, \mu, \tau$, with background matter in the flat space-time is described by the following effective Lagrangian 5 :

$$
\mathcal{L}_{\text {eff }}=-\sum_{\alpha} \bar{\nu}_{\alpha} \gamma_{\mu}^{\mathrm{L}} \nu_{\alpha} \cdot f_{\alpha}^{\mu}
$$

where $\gamma_{\mu}^{\mathrm{L}}=\gamma_{\mu}\left(1-\gamma^{5}\right) / 2, \gamma^{\mu}=\left(\gamma^{0}, \gamma\right)$ are the Dirac matrices, and $\gamma^{5}=\mathrm{i} \gamma^{0} \gamma^{1} \gamma^{2} \gamma^{3}$.

The interaction Lagrangian in Eq. (1) is derived in the mean field approximation using the effective external currents $f_{\alpha}^{\mu}$ depending on the characteristics of background matter as ${ }^{6}$

$$
f_{\alpha}^{\mu}=\sqrt{2} G_{\mathrm{F}} \sum_{f}\left(q_{\alpha, f}^{(1)} j_{f}^{\mu}+q_{\alpha, f}^{(2)} \lambda_{f}^{\mu}\right)
$$

where $G_{\mathrm{F}}$ is the Fermi constant and the sum is taken over all background fermions $f$. Here

$$
j_{f}^{\mu}=n_{f} u_{f}^{\mu},
$$


is the hydrodynamic current and

$$
\lambda_{f}^{\mu}=n_{f}\left(\left(\boldsymbol{\zeta}_{f} \mathbf{u}_{f}\right), \boldsymbol{\zeta}_{f}+\frac{\mathbf{u}_{f}\left(\boldsymbol{\zeta}_{f} \mathbf{u}_{f}\right)}{1+u_{f}^{0}}\right),
$$

is the four vector of the matter polarization. In Eqs. (3) and (41), $n_{f}$ is the invariant number density (the density in the rest frame of fermions), $\boldsymbol{\zeta}_{f}$ is the invariant polarization (the polarization in the rest frame of fermions), and $u_{f}^{\mu}=\left(u_{f}^{0}, \mathbf{u}_{f}\right)$ is the four velocity. To derive Eqs. (2)-(4) it is crucial that background fermions have constant velocity. Only in this situation one can make a boost to the rest frame of the fermions where $n_{f}$ and $\boldsymbol{\zeta}_{f}$ are defined. The explicit form of the coefficients $q_{\alpha, f}^{(1,2)}$ in Eq. (2) can be found in Ref. 6 .

Nowadays it is experimentally confirmed that the flavor neutrino eigenstates are the superposition of the neutrino mass eigenstates, $\psi_{i}, i=1,2, \ldots$,

$$
\nu_{\alpha}=\sum_{i} U_{\alpha i} \psi_{i}
$$

where $\left(U_{\alpha i}\right)$ is the unitary mixing matrix. The transformation in Eq. (5) diagonalizes the neutrino mass matrix. Only using the neutrino mass eigenstates we can reveal the nature of neutrinos, i.e. say whether they are Dirac or Majorana particles. Despite the great experimental efforts to shed light upon the nature of neutrinos, this issue still remains open. Here we shall suppose that $\psi_{i}$ correspond to Dirac fields.

The effective Lagrangian for the interaction of $\psi_{i}$ with background matter can be obtained using Eqs. (11) and (5),

$$
\mathcal{L}_{\text {eff }}=-\sum_{i j} \bar{\psi}_{i} \gamma_{\mu}^{\mathrm{L}} \psi_{j} \cdot g_{i j}^{\mu}
$$

where

$$
g_{i j}^{\mu}=\sum_{\alpha} U_{\alpha i}^{*} U_{\alpha j} f_{\alpha}^{\mu}
$$

is the nondiagonal effective potential in the mass eigenstates basis.

Using Eq. (6) one obtains that the corresponding Dirac equations for the neutrino mass eigenstates are coupled,

$$
\left[\mathrm{i} \gamma^{\mu} \partial_{\mu}-m_{i}-\gamma_{\mu}^{\mathrm{L}} g_{i i}^{\mu}\right] \psi_{i}=\sum_{j \neq i} \gamma_{\mu}^{\mathrm{L}} g_{i j}^{\mu} \psi_{j},
$$

where $m_{i}$ is the mass of $\psi_{i}$. One can proceed in the analytical analysis of Eq. (8) if we exactly account for only the diagonal effective potentials $g_{i i}^{\mu}$. To take into account the r.h.s. of Eq. (8), depending on the nondiagonal elements of the matrix $\left(g_{i j}^{\mu}\right)$, with $i \neq j$, one should apply a perturbative method (see Sec. 4 below). 


\section{Massive neutrinos in noninertial frames}

In this section we generalize the Dirac equation for a neutrino interacting with a background matter to the situation when the velocity of the matter motion is not constant. In particular, we study the case of the matter rotation with a constant angular velocity. Then we obtain the solution of the Dirac equation and find the energy spectrum.

If we discuss a neutrino mass eigenstate propagating in a nonuniformly moving matter, the expressions for $f_{\alpha}^{\mu}$ in Eqs. (2)-(4) become invalid since they are derived under the assumption of the unbroken Lorentz invariance. The most straightforward way to describe the neutrino evolution in matter moving with an acceleration is to rewrite the Dirac equation for a neutrino in the noninertial frame where matter is at rest. In this case one can unambiguously define the components of $f_{\alpha}^{\mu}$. Assuming that background fermions are unpolarized, we find that in this reference frame

$$
f_{\alpha}^{0}=\sqrt{2} G_{\mathrm{F}} \sum_{f} q_{\alpha, f}^{(1)} n_{f} \neq 0,
$$

with the rest of the effective potentials being equal to zero.

It is known that the motion of a test particle in a noninertial frame is equivalent to the interaction of this particle with a gravitational field. The Dirac equation for a massive neutrino moving in a curved space-time and interacting with background matter can be obtained by the generalization of Eq. (8) (see also Ref. 7),

$$
\left[\mathrm{i} \gamma^{\mu}(x) \nabla_{\mu}-m\right] \psi=\frac{1}{2} \gamma_{\mu}(x) g^{\mu}\left[1-\gamma^{5}(x)\right] \psi,
$$

where $\gamma_{\mu}(x)$ are the coordinate dependent Dirac matrices, $\nabla_{\mu}=\partial_{\mu}+$ $\Gamma_{\mu}$ is the covariant derivative, $\Gamma_{\mu}$ is the spin connection, $\gamma^{5}(x)=$ $-\frac{\mathrm{i}}{4 !} E^{\mu \nu \alpha \beta} \gamma_{\mu}(x) \gamma_{\nu}(x) \gamma_{\alpha}(x) \gamma_{\beta}(x), E^{\mu \nu \alpha \beta}=\frac{1}{\sqrt{-g}} \varepsilon^{\mu \nu \alpha \beta}$ is the covariant antisymmetric tensor in curved space-time, and $g=\operatorname{det}\left(g_{\mu \nu}\right)$ is the determinant of the metric tensor $g_{\mu \nu}$. Note that in Eq. (10) we account for only the diagonal neutrino interaction with matter. That is why we omit the index $i$ in order not to encumber the notation: $m \equiv m_{i}$ etc. It should be noted that analogous Dirac equation was discussed in Ref. 8.

We shall be interested in the neutrino motion in matter rotating with the constant angular velocity $\omega$. Choosing the corotating frame we get that only $g^{0} \equiv g_{i i}^{0}$ is nonvanishing, cf. Eqs. (7) and (9).

\subsection{Neutrino motion in a rotating frame}

The interval in the rotating frame is $\frac{9}{}$

$$
\mathrm{d} s^{2}=g_{\mu \nu} \mathrm{d} x^{\mu} \mathrm{d} x^{\nu}=\left(1-\omega^{2} r^{2}\right) \mathrm{d} t^{2}-\mathrm{d} r^{2}-2 \omega r^{2} \mathrm{~d} t \mathrm{~d} \phi-r^{2} \mathrm{~d} \phi^{2}-\mathrm{d} z^{2},
$$

where we use the cylindrical coordinates $x^{\mu}=(t, r, \phi, z)$. One can check that the metric tensor in Eq. (11) can be diagonalized, $\eta_{a b}=e_{a}^{\mu} e_{b}^{\nu} g_{\mu \nu}$, if we use the following 
vierbein vectors:

$$
\begin{aligned}
e_{0}^{\mu} & =\left(\frac{1}{\sqrt{1-\omega^{2} r^{2}}}, 0,0,0\right), \\
e_{1}^{\mu} & =(0,1,0,0), \\
e_{2}^{\mu} & =\left(\frac{\omega r}{\sqrt{1-\omega^{2} r^{2}}}, 0, \frac{\sqrt{1-\omega^{2} r^{2}}}{r}, 0\right), \\
e_{3}^{\mu} & =(0,0,0,1) .
\end{aligned}
$$

Here $\eta_{a b}=\operatorname{diag}(1,-1,-1,-1)$ is the metric in a locally Minkowskian frame.

Let us introduce the Dirac matrices in a locally Minkowskian frame by $\gamma^{\bar{a}}=$ $e^{a}{ }_{\mu} \gamma^{\mu}(x)$, where $e^{a}{ }_{\mu}$ is the inverse vierbein: $e^{a}{ }_{\mu} e_{b}{ }^{\mu}=\delta_{b}^{a}$. Starting from now, we shall mark an index with a bar to demonstrate that a gamma matrix is defined in a locally Minkowskian frame. As shown in Ref. [4, $\gamma^{5}(x)=\mathrm{i} \gamma^{\overline{0}} \gamma^{\overline{1}} \gamma^{\overline{2}} \gamma^{\overline{3}}=\gamma^{\overline{5}}$ does not depend on coordinates.

After the straightforward calculation of the spin connection on the basis of Eq. (12), the Dirac Eq. (10) can be rewritten as

$$
\begin{aligned}
{[\mathcal{D}-m] \psi=\frac{1}{2} \sqrt{1-\omega^{2} r^{2}} \gamma^{\overline{0}} g^{0}\left(1-\gamma^{\overline{5}}\right) \psi, } \\
\mathcal{D}=\mathrm{i} \frac{\gamma^{\overline{0}}+\omega r \gamma^{2}}{\sqrt{1-\omega^{2} r^{2}}} \partial_{0}+\mathrm{i} \gamma^{\overline{1}}\left(\partial_{r}+\frac{1}{2 r}\right)+\mathrm{i} \gamma^{\overline{2}} \frac{\sqrt{1-\omega^{2} r^{2}}}{r} \partial_{\phi}+\mathrm{i} \gamma^{\overline{3}} \partial_{z} \\
\quad-\frac{\omega}{2\left(1-\omega^{2} r^{2}\right)} \gamma^{\overline{3}} \gamma^{\overline{5}} .
\end{aligned}
$$

The analogous Dirac equation was recently derived in Ref. 10. Since Eq. (13) does not explicitly contain $t, \phi$, and $z$, its solution can be expressed as

$$
\psi=\exp \left(-\mathrm{i} E t+\mathrm{i} J_{z} \phi+\mathrm{i} p_{z} z\right) \psi_{r},
$$

where $\psi_{r}=\psi_{r}(r)$ is the spinor depending on the radial coordinate, $J_{z}=\frac{1}{2}-l$ (see, e.g., Ref. 11), and $l=0, \pm 1, \pm 2, \ldots$.

In Eq. (13) one can neglect terms $\sim(\omega r)^{2}$. Indeed, if we study a neutrino in a rotating pulsar, then $r \lesssim 10 \mathrm{~km}$ and $\omega \lesssim 10^{3} \mathrm{~s}^{-1}$. Thus $(\omega r)^{2} \lesssim 1.1 \times 10^{-3}$ is a small parameter. Therefore Eq. (13) can be transformed to

$$
\begin{aligned}
{\left[\mathrm{i} \gamma^{\overline{1}}\left(\partial_{r}+\frac{1}{2 r}\right)-\gamma^{2}\left(\frac{J_{z}}{r}-\omega r E\right)+\gamma^{\overline{0}}\right.} & \left(E-\frac{g^{0}}{2}\right)-\gamma^{\overline{3}} p_{z} \\
& \left.+\frac{g^{0}}{2} \gamma^{\overline{0}} \gamma^{\overline{5}}-\frac{\omega}{2} \gamma^{\overline{3}} \gamma^{\overline{5}}-m\right] \psi_{r}=0
\end{aligned}
$$

where we keep only the terms linear in $\omega$. It should be noted that the term $\sim \omega \gamma^{\overline{3}} \gamma^{\overline{5}}$ in Eq. (15) is equivalent to the neutrino interaction with matter moving with an effective velocity. 
The solution of Eq. (15) can be presented in the form $\stackrel{4}{4}, \psi_{r}^{\mathrm{L}}=(0, \eta)^{\mathrm{T}}$ and $\psi_{r}^{\mathrm{R}}=(\xi, 0)^{\mathrm{T}}$, where

$$
\eta=\left(\begin{array}{c}
-\mathrm{i} C_{1} I_{N, s} \\
C_{2} I_{N-1, s}
\end{array}\right), \quad \xi=\left(\begin{array}{c}
C_{3} I_{N, s} \\
-\mathrm{i} C_{4} I_{N-1, s}
\end{array}\right) .
$$

Here $N=0,1,2, \ldots, s=N-l, I_{N, s}=I_{N, s}(\rho)$ is the Laguerre function, and $\rho=E \omega r^{2}$. The explicit form of the Laguerre function can be found, e.g., in Ref. 4. To derive Eq. (16) we use the Dirac matrices in the chiral representation 12 .

In the important case when $\omega \ll g^{0}$, the coefficients $C_{i}, i=1, \ldots, 4$, in Eq. (16) are expressed in the following way $\stackrel{4}{=}$ :

$$
\begin{array}{ll}
C_{1}^{2} \approx \frac{E_{A} \omega}{2 \pi} \frac{E_{A}-p_{z}-g^{0}}{E_{A}-g^{0}}, & C_{3}^{2} \approx \frac{\omega}{2 \pi}\left(E_{S}+p_{z}\right), \\
C_{2}^{2} \approx \frac{E_{A} \omega}{2 \pi} \frac{E_{A}+p_{z}-g^{0}}{E_{A}-g^{0}}, & C_{4}^{2} \approx \frac{\omega}{2 \pi}\left(E_{S}-p_{z}\right) .
\end{array}
$$

It should be noted that the solutions presented in Eqs. (16) and (17) satisfy the normalization condition,

$$
\int \psi_{N, s, p_{z}}^{\dagger}(x) \psi_{N^{\prime}, s^{\prime}, p_{z}^{\prime}}(x) \sqrt{-g} \mathrm{~d}^{3} x=\delta_{N N^{\prime}} \delta_{s s^{\prime}} \delta\left(p_{z}-p_{z}^{\prime}\right) .
$$

Here $\psi$ and $\psi_{r}$ are related by Eq. (14).

The energy levels in Eq. (17) are

$$
\begin{aligned}
{\left[E_{A}-2 N \omega-g^{0}\right]^{2} } & =(2 N \omega)^{2}+4 N \omega g^{0}+\left(p_{z}-\frac{\omega}{2}\right)^{2}, \\
{\left[E_{S}-2 N \omega\right]^{2} } & =(2 N \omega)^{2}+\left(p_{z}+\frac{\omega}{2}\right)^{2},
\end{aligned}
$$

where $E_{A}$ and $E_{S}$ are the energies of active and sterile neutrinos respectively. Comparing the expression for $E_{S} \approx 2 N \omega+\sqrt{(2 N \omega)^{2}+p_{z}^{2}}$ with the energy of a neutrino in an inertial nonrotating frame $\sqrt{\mathbf{p}_{\perp}^{2}+p_{z}^{2}}$, where $\mathbf{p}_{\perp}$ is the momentum in the equatorial plane, we can identify $2 N \omega$ inside the square root as $\left|\mathbf{p}_{\perp}\right|$. It should be also noted that the term $2 N \omega$, which additively enters to both $E_{A}$ and $E_{S}$, is due to the noninertial effects for a Dirac fermion in a rotating frame $\underline{\underline{13}}$.

We can also get the corrections to the energy levels due to the nonzero mass, $E_{A, S} \rightarrow E_{A, S}+E_{A, S}^{(1)}$. On the basis of Eq. (16) and (17) one finds the expression for $E_{A, S}^{(1)}$ in the limit $\omega \ll g^{0}$,

$$
E_{A}^{(1)}=\frac{m^{2}}{2\left(E_{A}-2 N \omega-g^{0}\right)}, \quad E_{S}^{(1)}=\frac{m^{2}}{2\left(E_{S}-2 N \omega\right)} .
$$

If we discuss neutrinos moving along the rotation axis, then $2 N \omega \ll\left|p_{z}\right|$. Using Eq. (19) we get the energy levels of active neutrinos in this case

$$
E_{A}=\left|p_{z}\right|+g^{0}\left(1+\frac{2 N \omega}{\left|p_{z}\right|}\right)+2 N \omega+\frac{2(N \omega)^{2}}{\left|p_{z}\right|}+\frac{m^{2}}{2\left|p_{z}\right|},
$$


where we also keep the mass correction in Eq. (20). One can see in Eq. (21) that $\left|p_{z}\right|+g^{0}+\frac{m^{2}}{2\left|p_{z}\right|}$ corresponds to the energy of a left-handed neutrino interacting with background matter in a flat space-time. The rest of the terms in Eq. (21) are the corrections due to the matter rotation.

\section{Flavor oscillations of Dirac neutrinos in rotating matter}

In this section we study the evolution of the system of massive mixed neutrinos in rotating matter. We formulate the initial condition for this system and derive the effective Schrödinger equation which governs neutrino flavor oscillations. Then we find the correction to the resonance condition owing to the matter rotation and estimate its value for a millisecond pulsar.

We can generalize the results of Sec. 3 to include different neutrino eigenstates. The interaction of neutrino mass eigenstates with background matter is nondiagonal, cf. Eq. (6). Therefore the generalization of Eq. (13) for several mass eigenstates $\psi_{i}$ reads

$$
\left[\mathcal{D}-m_{i}\right] \psi_{i}=\frac{1}{2} \gamma^{\overline{0}} g_{i}^{0}\left(1-\gamma^{\overline{5}}\right) \psi_{i}+\frac{1}{2} \gamma^{\overline{0}} \sum_{j \neq i} g_{i j}^{0}\left(1-\gamma^{\overline{5}}\right) \psi_{j},
$$

where $g_{i}^{0} \equiv g_{i i}^{0}$ and $g_{i j}^{0}$ are the time components of the matrix $\left(g_{i j}^{\mu}\right)$ given in Eq. (7), $m_{i}$ is the mass of $\psi_{i}$, and $\mathcal{D}$ can be found in Eq. (13). As in Sec. 3. we omitted the term $(\omega r)^{2} \ll 1$ in Eq. (22). Note that Eq. (22) is a generalization of Eq. (8) for a system of the neutrino mass eigenstates moving in a rotating frame.

We shall study the evolution of active ultrarelativistic neutrinos and neglect neutrino-antineutrino transitions. In this case we can restrict ourselves to the analysis of two component spinors. The general solution of Eq. (22) has the form,

$$
\eta_{i}(x)=\sum_{N, s} \int \frac{\mathrm{d} p_{z}}{\sqrt{2 \pi}} a_{N, s, p_{z}}^{(i)} e^{\mathrm{i} p_{z} z+\mathrm{i} J_{z} \phi} u_{N, s, p_{z}}(r) e^{-\mathrm{i} E_{i} t},
$$

where $u_{N, s, p_{z}}$ are the basis spinors and $a_{N, s, p_{z}}^{(i)}=a_{N, s, p_{z}}^{(i)}(t)$ are the $c$-number functions. The energy levels $E_{i}$ are given in Eq. (21) with $m \rightarrow m_{i}$. Here we omit the subscript $A$ in order not to encumber the notation. Our goal is to find the coefficient $a_{N, s, p_{z}}^{(i)}=a_{N, s, p_{z}}^{(i)}(t)$. We neglect the small ratio $\omega / g_{i}^{0}$ in Eq. (23).

Considering the system of two neutrino mass eigenstates, $i=1,2$, parameterized with one mixing angle $\theta$, and choosing the appropriate initial condition ${ }^{4}$, on the basis of Eq. (22) we get the effective Schrödinger equation for $\tilde{\Psi}^{\mathrm{T}}=\left(a_{1}, a_{2}\right)$,

$$
\mathrm{i} \frac{\mathrm{d} \tilde{\Psi}}{\mathrm{d} t}=\left(\begin{array}{cc}
0 & g_{12}^{0} \exp \left[\mathrm{i}\left(E_{1}-E_{2}\right) t\right] \\
g_{12}^{0} \exp \left[\mathrm{i}\left(E_{2}-E_{1}\right) t\right] & 0
\end{array}\right) \tilde{\Psi} .
$$

Here we omitted all the indexes of $a_{i}$ besides $i=1,2$. It is convenient to introduce the modified effective wave function $\Psi=\mathcal{U}_{3} \tilde{\Psi}$, where $\mathcal{U}_{3}=\operatorname{diag}\left(e^{\mathrm{i} \Omega t / 2}, e^{-\mathrm{i} \Omega t / 2}\right)$, $\Omega=E_{1}-E_{2}$. Using Eq. (24), we get for $\Psi$

$$
\mathrm{i} \frac{\mathrm{d} \Psi}{\mathrm{d} t}=\left(\begin{array}{cc}
\Omega / 2 & g_{12}^{0} \\
g_{12}^{0} & -\Omega / 2
\end{array}\right) \Psi .
$$


Note that Eq. (25) has the form of the effective Schrödinger equation one typically deals with in the study of neutrino flavor oscillations in background matter.

If the transition probability for $\nu_{\alpha} \leftrightarrow \nu_{\beta}$ is close to one, i.e. $P_{\nu_{\beta} \rightarrow \nu_{\alpha}}=$ $\left|\left\langle\nu_{\alpha}(t) \mid \nu_{\beta}(0)\right\rangle\right|^{2} \approx 1$, flavor oscillations of neutrinos are said to be at resonance. Using Eqs. (5), (9), (21), and (25), the resonance condition can be written as,

$$
\left(f_{\alpha}^{0}-f_{\beta}^{0}\right)\left(1+\frac{2 N \omega}{\left|p_{z}\right|}\right)+\frac{\Delta m^{2}}{2\left|p_{z}\right|} \cos 2 \theta=0,
$$

where $\Delta m^{2}=m_{1}^{2}-m_{2}^{2}$ is the mass squared difference.

Let us consider electroneutral background matter composed of electrons, protons, and neutrons. If we study the $\nu_{e} \rightarrow \nu_{\alpha}$ oscillation channel, where $\alpha=\mu, \tau$, we get that $f_{\nu_{\alpha}}^{0}=-\frac{1}{\sqrt{2}} G_{\mathrm{F}} n_{n}$ and $f_{\nu_{\beta}}^{0} \equiv f_{\nu_{e}}^{0}=\sqrt{2} G_{\mathrm{F}}\left(n_{e}-\frac{1}{2} n_{n}\right)$, where $n_{e}$ and $n_{n}$ are the densities of electrons and neutrons. Using Eq. (26), we obtain that

$$
\sqrt{2} G_{\mathrm{F}} n_{e}\left(1+\frac{2 N \omega}{\left|p_{z}\right|}\right)=\frac{\Delta m^{2}}{2\left|p_{z}\right|} \cos 2 \theta .
$$

At the absence of rotation, $\omega=0$, Eq. (27) is equivalent to the Mikheyev-SmirnovWolfenstein resonance condition in background matter 14 .

Let us evaluate the contribution of the matter rotation to the resonance condition in Eq. (27) for a neutrino emitted inside a rotating pulsar. We make a natural assumption that for a corotating observer neutrinos are emitted in a spherically symmetric way from a neutrinosphere. That is we should take that $l \approx 0$ and $N \approx s$. Then the trajectory of a neutrino is deflected because of the noninertial effects and the interaction with background matter. The radius $\mathcal{R}$ of the trajectory can be found from

$$
\mathcal{R}^{2}=2\left|p_{z}\right| \omega \int_{0}^{\infty} r^{2}\left|u_{N, s, p_{z}}(r)\right|^{2} r \mathrm{~d} r \approx \frac{2 N}{\left|p_{z}\right| \omega},
$$

where we take into account that $N \gg 1$.

We shall assume that $\mathcal{R} \sim R_{0}$, where $R_{0}=10 \mathrm{~km}$ is the pulsar radius. In this case neutrinos escape a pulsar. Taking that $\omega=10^{3} \mathrm{~s}^{-1}$ and using Eq. (28), we get that the correction to the resonance condition in Eq. [27] is $\frac{2 N \omega}{\left|p_{z}\right|} \approx\left(R_{0} \omega\right)^{2} \approx 10^{-3}$. The obtained correction to the effective number density is small but nonzero. This result corrects our previous statement ${ }^{2}$ that a matter rotation does not contribute neutrino flavor oscillations.

\section{Conclusion}

In conclusion we notice that we have studied the evolution of massive mixed neutrinos in nonuniformly moving background matter. The interaction of neutrinos with background fermions is described in frames of the Fermi theory (see Sec. 2). A particular case of the matter rotating with a constant angular velocity has been studied in Sec. 3.1. We have derived the Dirac equation for a weakly interacting 
neutrino in a rotating frame and found its solution in case of ultrarelativistic neutrinos, cf. Eqs. (16) and (17). The energy spectrum obtained in Eqs. (19) and (20) includes the correction owing to the nonzero neutrino mass.

We have used the Dirac equation in a noninertial frame, cf. Eq. (10), as a main tool for the study of the neutrino motion in matter moving with an acceleration. To develop the quantum mechanical description of such a neutrino we have chosen a noninertial frame where matter is at rest. In this frame the effective potential of the neutrino-matter interaction is well defined. However, the wave equation for a neutrino turns out to be more complicated since one has to deal with noninertial effects.

In Sec. 4 we have generalized our results to include various neutrino generations as well as mixing between them. We have derived the effective Schrödinger equation which governs neutrino flavor oscillations. We have obtained the correction to the resonance condition in background matter owing to the matter rotation. Studying neutrino oscillations in a millisecond pulsar, we have obtained that the effective number density changes by $0.1 \%$ owing to the matter rotation.

Despite the obtained correction is small, we may suggest that our results can have some implication to the explanation of great linear velocities of pulsars. It was suggested in Ref. 15 that an asymmetry in neutrino oscillations in a magnetized pulsar can explain a great linear velocity of the compact star. An evidence for the alignment of the angular and the linear velocity vectors of pulsars was reported in Ref. 16. Therefore we may suggest that neutrino flavor oscillations in a rapidly rotating pulsar can contribute to its linear velocity. It should be noted that neutrino spin-flavor oscillations, including noninertial effects, in a rapidly rotating magnetized star were studied in Ref. 17 in the context of the explanation of high linear velocities of pulsars.

Finally, we mention that the Dirac equation for a fermion, electroweakly interacting with the rotating background matter, was recently solved 18 . The vierbein vectors, different from these in Eq. (12), were used in Ref. 18. Comparing the energy levels obtained in Ref. 18 with the results of the general analysis 13 , one concludes that the vierbein used in Ref. 18 is more appropriate for the description of ultrarelativistic particles like neutrinos.

\section{Acknowledgments}

I am thankful to the organizers of the International Conference on Massive Neutrinos for the invitation and support, to S. P. Gavrilov for helpful comments, to FAPESP (Brazil) for the Grant No. 2011/50309-2, to the Tomsk State University Competitiveness Improvement Program and to RFBR (research project No. 15-0200293) for partial support. 


\section{References}

1. G. Lambiase, Neutrino oscillations in non-inertial frames and the violation of the equivalence principle. Neutrino mixing induced by the equivalence principle violation, Eur. Phys. J. C 19, 553 (2001).

2. M. Dvornikov and C. O. Dib, Spin-down of neutron stars by neutrino emission, Phys. Rev. D 82, 043006 (2010) arXiv:0907.1445.

3. B. Basu and D. Chowdhury, Inertial effect on spin orbit coupling and spin transport, Ann. Phys. (N.Y.) 335, 47 (2013) arXiv:1302.1063.

4. M. Dvornikov, Neutrino interaction with matter in a noninertial frame, J. High Energy Phys. 10 (2014) 053 arXiv:1408.2735.

5. C. Giunti and C. W. Kim, Fundamentals of Neutrino Physics and Astrophysics (Oxford University Press, Oxford, 2007), pp. 137-179.

6. M. Dvornikov and A. Studenikin, Neutrino spin evolution in presence of general external fields, J. High Energy Phys. 09 (2002) 016 hep-ph/0202113.

7. A. A. Grib, S. G. Mamaev and V. M. Mostepanenko, Quantum Effects in Intense External Fields: Methods and Results not Related to the Perturbation Theory (Atomizdat, Moscow, 1980), pp. 13-15.

8. D. Píriz, M. Roy and J. Wudka, Neutrino oscillations in strong gravitational fields, Phys. Rev. D 54, 1587 (1996) hep-ph/9604403.

9. L. D. Landau and E. M. Lifshitz, The Classical Theory of Fields (Butterworth Heinemann, Amsterdam, 1994), 4th ed., pp. 329-330.

10. K. Bakke, Rotating effects on the Dirac oscillator in the cosmic string spacetime, Gen. Relativ. Grav. 45, 1845 (2013) arXiv:1307.2847.

11. P. Schluter, K.-H. Wietschorke and W. Greiner, The Dirac equation in orthogonal coordinate systems: I. The local representation, J. Phys. A: Math. Gen. 16, 1999 (1983).

12. C. Itzykson and J.-B. Zuber, Quantum Field Theory (McGraw-Hill, New York, 1980), pp. 691-696.

13. F. W. Hehl and W.-T. Ni, Inertial effects of a Dirac particle, Phys. Rev. D 42, 2045 (1990).

14. M. Blennow and A. Yu. Smirnov, Neutrino propagation in matter, Adv. High Energy Phys. 2013, 972485 (2013) arXiv:1306.2903.

15. A. Kusenko and G. Segrè, Velocities of pulsars and neutrino oscillations, Phys. Rev. Lett. 77, 4872 (1996) hep-ph/9606428.

16. S. Johnston, G. Hobbs, S. Vigeland, M. Kramer, J. M. Weisberg and A. G. Lyne, Evidence for alignment of the rotation and velocity vectors in pulsars, Mon. Not. Roy. Astron. Soc. 364, 1397 (2005) astro-ph/0510260.

17. G. Lambiase, Pulsar kicks induced by spin flavor oscillations of neutrinos in gravitational fields, Mon. Not. Roy. Astron. Soc. 362, 867 (2005) [astro-ph/0411242].

18. M. Dvornikov, Galvano-rotational effect in a pulsar induced by the electroweak interaction, J. Cosmol. Astropart. Phys. 05 (2015) 037 arXiv:1503.00608. 